\title{
Surfactant Activated Dip-Pen Nanolithography
}

\author{
Hyungil Jung, Chiraj K. Dalal, Steven Kuntz, Raman Shah and C. Patrick Collier*
}

Division of Chemistry and Chemical Engineering, California Institute of Technology, Pasadena, CA 91125

\section{Supporting Information}

\section{Fluorescence Assay for Enzymatic Activity from DPN-Written Arrays of Horseradish Peroxidase:}

As a proof of principle showing that enzymes can be patterned on glass surfaces with DPN without loss of functionality, we immobilized avidin-linked horseradish peroxidase (HRP) to maleimide $\mathrm{PEO}_{2}$ biotin-written areas on MPTMS-silanized glass and directly characterized enzymatic activity from the sites with a fluorescence-based assay involving the conversion of fluorogenic substrate molecules to fluorescent products. We used DPN to write four $1 \mathrm{x} 1 \mu \mathrm{m}$ regions of biotin on silanized glass, separated by $30 \mu \mathrm{m}$ in a square array. After passivation of the unwritten areas of the MPTMS surface with PEGmaleimide, incubation with HRP-avidin, and washing steps, we added one $40 \mu \mathrm{L}$ drop of an aqueous solution consisting of $40 \mu \mathrm{M}$ amplex red and $800 \mu \mathrm{M}$ hydrogen peroxide onto the patterned glass coverslip with a micropipette, while monitoring fluorescence with an epi-fluorescence microscope. Images were captured by a high-resolution CCD camera every 200 msec. Frame A in Figure S1 shows the probable locations of the written areas. The camera started capturing images as soon as the drop was delivered to the surface. It was difficult to define a sharp starting time for this reaction, however, since we could not characterize the effects that convective mixing had on the concentration profile of the reagents after the drop was pipetted on the glass. The areas of the fluorescent regions merged much 
faster than would be expected by simple molecular diffusion, which should be about 7 sec (assuming a diffusion coefficient of $66 \mathrm{micron}^{2} / \mathrm{sec}$ for resorufin and a diffusion distance of 30 microns). Nevertheless, the multiple array sites of peroxidase enzymes patterned with DPN all showed similar pseudo-first-order reaction kinetics, consistent with a Michaelis-Menten mechanism.
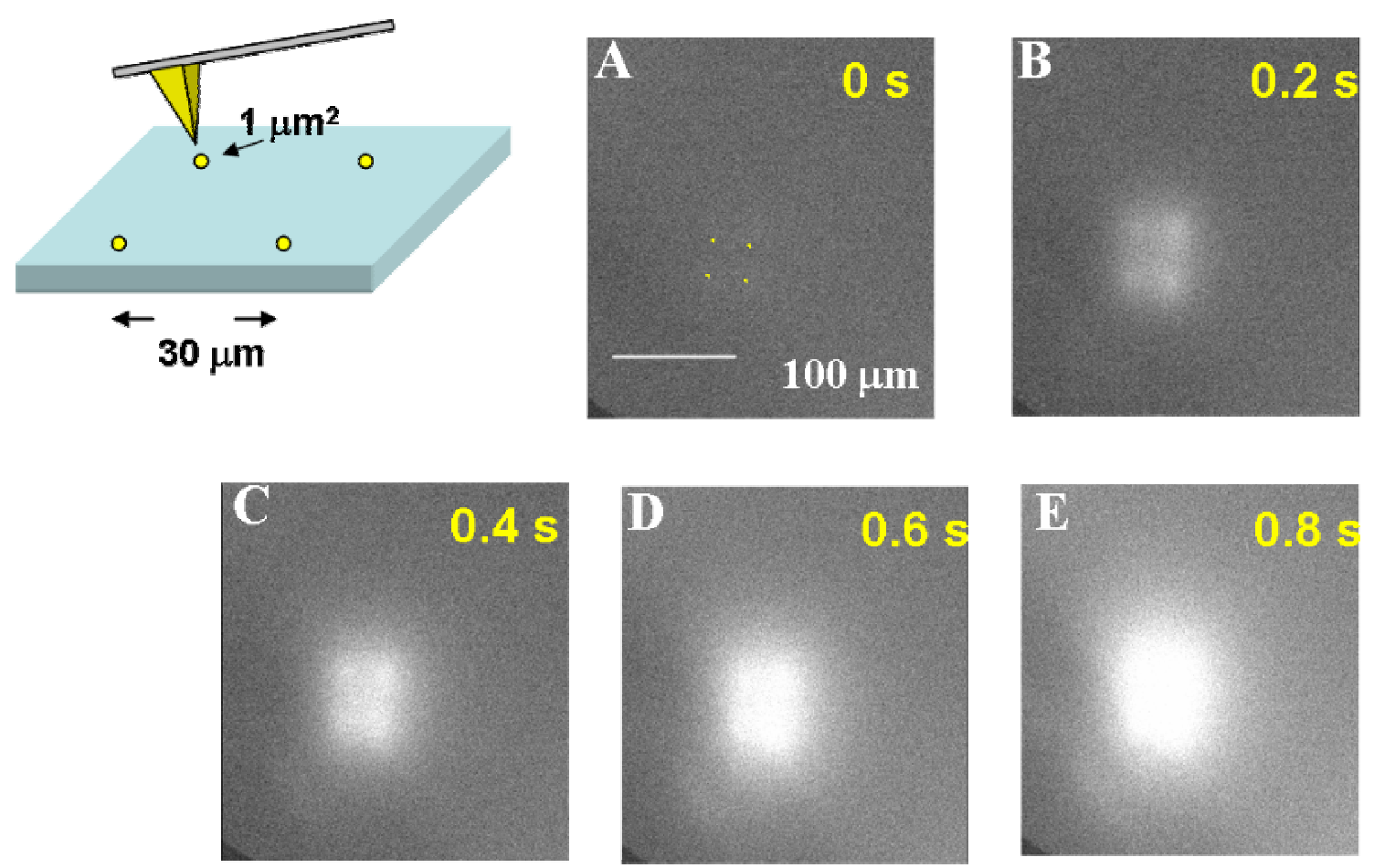

Figure S1. Enzymatic conversion of fluorogenic substrate molecules (amplex red) to fluorescent products (resorufin). Images of fluorescent products were taken after the times indicated in each frame.

\section{Correlations between $\mathrm{RH}$ and Tween-20 concentrations needed for activation of biotin writing:}

Table 1 shows the minimum RH needed to observe biotin deposition onto MPTMS for Tween-20 concentrations ranging from $0.005 \%$ to $0.1 \%$; also included are the minimum tip-substrate contact times at which biotin deposition was observed. These delay times have large uncertainties, since we could not continuously monitor for initiation of ink deposition in real time. In all cases, the minimum RH needed for biotin writing scaled inversely with surfactant concentration. 
Table 1. Relative humidity-surfactant concentration correlations for biotin deposition with DPN.

\begin{tabular}{|l|l|l|}
\hline $\begin{array}{l}\text { Tween-20 } \\
(\% \mathrm{v} / \mathrm{v})\end{array}$ & Minimum RH & Contact time (s) \\
\hline $0.005 \%$ & $39 \%$ & 30 \\
\hline $0.01 \%$ & $30 \%$ & 60 \\
\hline $0.1 \%$ & $23 \%$ & 60 \\
\hline
\end{tabular}

A subset of the data in Table 1 is illustrated with Figure S2, which contains two LFM images that show the effect of increased Tween-20 concentration on DPN writing of biotin at 30\% RH. For Figure S2a, $0.005 \%$ Tween-20 was included in the ink, while for Figure S2b, the surfactant concentration was $0.01 \%$. Both images show the effects of increasing tip-substrate contact times for 1, 30, and 60 seconds. Figure S2b shows that the inclusion of $0.01 \%$ Tween-20 in the ink at 30\% RH resulted in large circular spots with dark contrast, indicative of decreased friction relative to the MPTMS background. We believe this change in contrast is due to preferential adsorption of water on MPTMS in the vicinity of the AFM tip; similar phenomena have been seen before in LFM images and have been attributed to the effects of water adsorption. ${ }^{1}$ It can be seen from Figure S2a that, at $0.005 \%$ Tween-20, this change in contrast is difficult to observe at the same $\mathrm{RH}(30 \%)$, suggesting that the transport of water to MPTMS was facilitated by the surfactant. The size of the dark spots increased rapidly with humidity, quickly reaching several tens of microns in diameter at $\mathrm{RH}$ values greater than $50 \%$, even for tip-substrate contact times of less than 1 second. At higher RH, we were no longer able to observe these dark circles with LFM, even at the highest tip scan speeds. We believe that this was because the affected area due to tip-induced water adsorption had exceeded our scanning field-of-view (10-30 $\mu \mathrm{m})$.

After one minute, small bright spots can be seen in the center of the two dark circles at the bottom of the image in Figure S2b, which are not seen in Figure S2a. We incubated a 2.5 x $10 \mu \mathrm{m}$ rectangle, prepared under the same conditions as the sample in Figure S2b, with Cy3-streptavidin. Fluorescence was only observed from the region of the pattern that corresponded to bright contrast in LFM, and not 
from the dark border (not shown). This indicates that only the regions with bright contrast contained immobilized biotin.
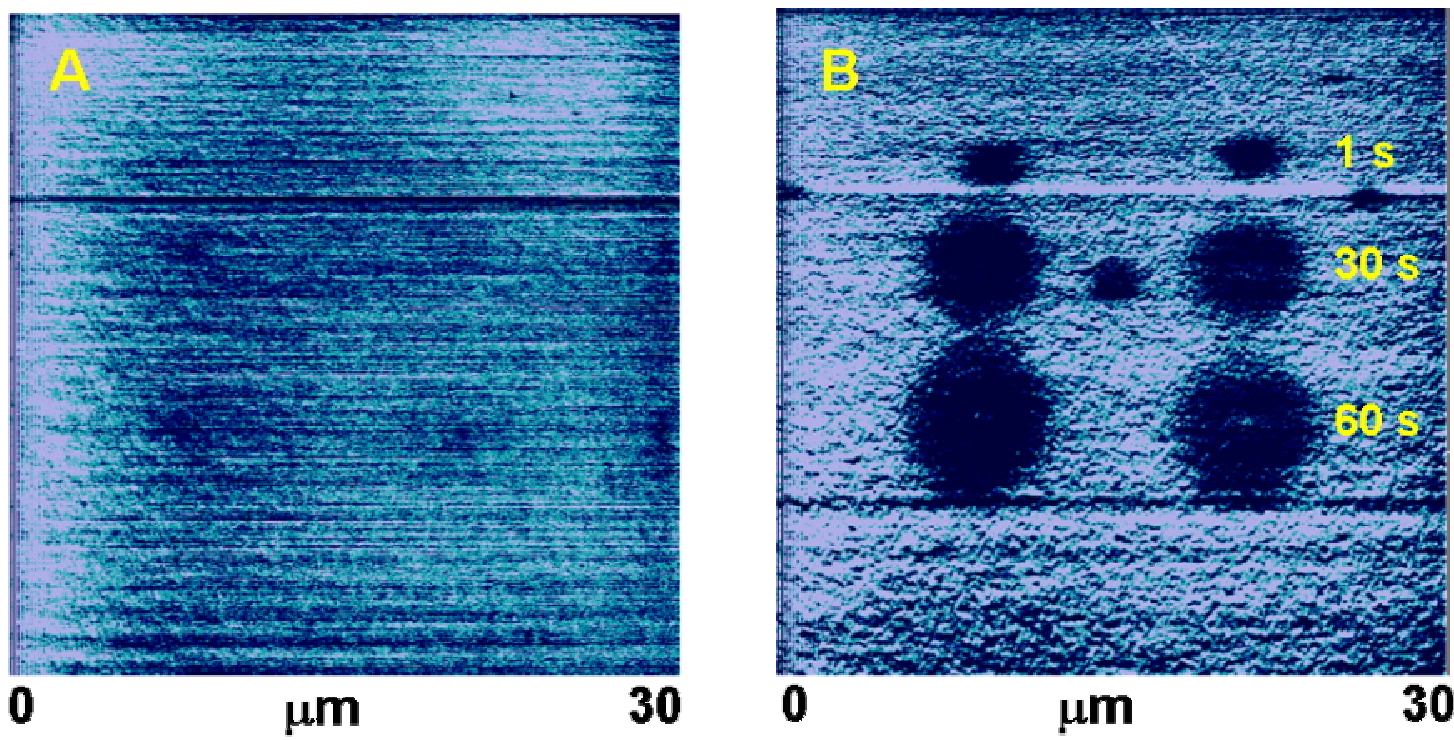

Figure S2. LFM images of DPN patterns on MPTMS-coated glass with (a) $0.005 \%$ Tween-20 and (b) 0.01\% Tween-20 included in the ink (with $25 \mathrm{mg} / \mathrm{mL}$ biotin), at 30\% RH. For both images, the AFM tip was in contact with the substrate for 1,30 , and 60 seconds.

\section{LFM images of biotin on clean glass and Tween-20 on MPTMS:}

Figure S3 shows LFM images from control experiments in which only biotin or surfactant, but not both, were included in the ink. Figure S3a shows $2 \mu \mathrm{m}$ long lines of biotin $(25 \mathrm{mg} / \mathrm{mL}$ in PBS) patterned on clean hydrophilic glass at $45 \% \mathrm{RH}$, with no Tween-20. Additional control experiments with silanized AFM tips used as is or dipped in PBS buffer solution did not show any patterns. Figure S3b shows circular patterns on MPTMS with 0.1\% Tween-20 included in the ink at 55\% RH for 5 sec, $10 \mathrm{sec}, 15 \mathrm{sec}$, and $25 \mathrm{sec}$ hold times. These patterns show that the surfactant was rapidly adsorbed onto MPTMS from the AFM tip. 

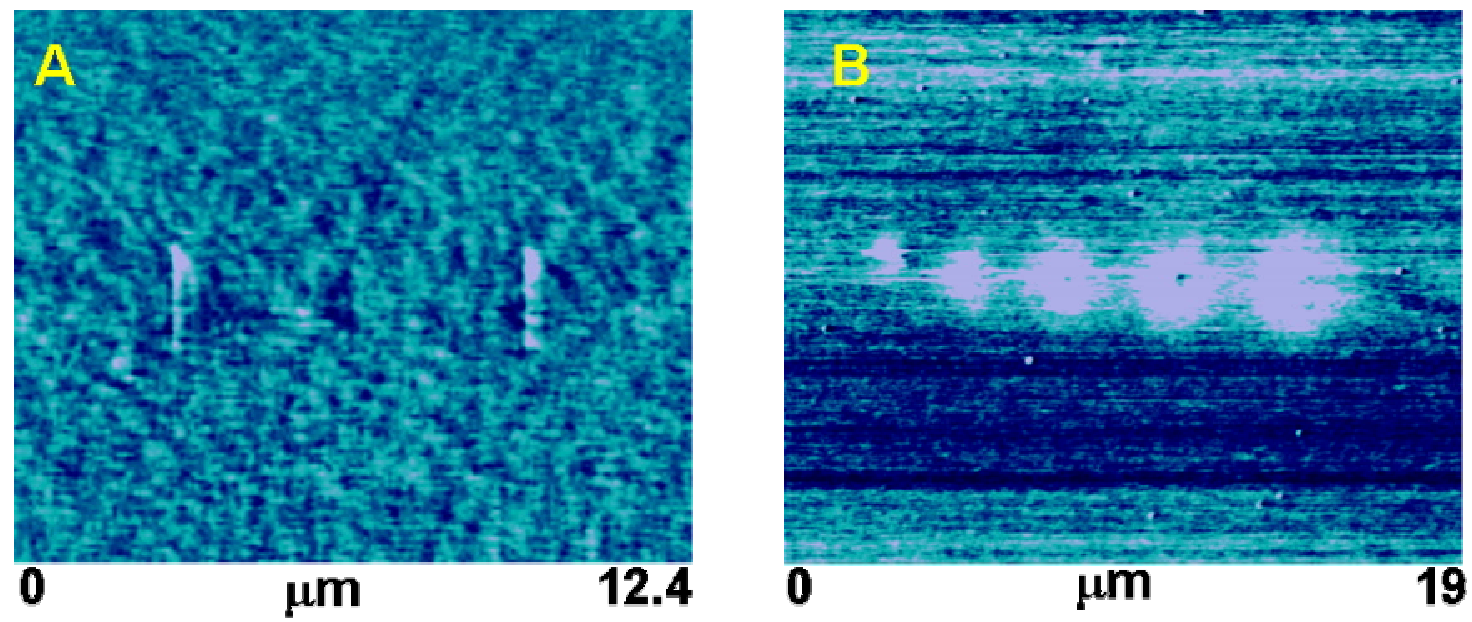

Figure S3. (a) LFM image of biotin on clean glass at 45\% RH. (b) LFM image of $0.1 \%$ Tween-20 on MPTMS at 55\% RH.

Tapping mode AFM images of Cy3-streptavidin conjugated to biotin patterned with DPN:
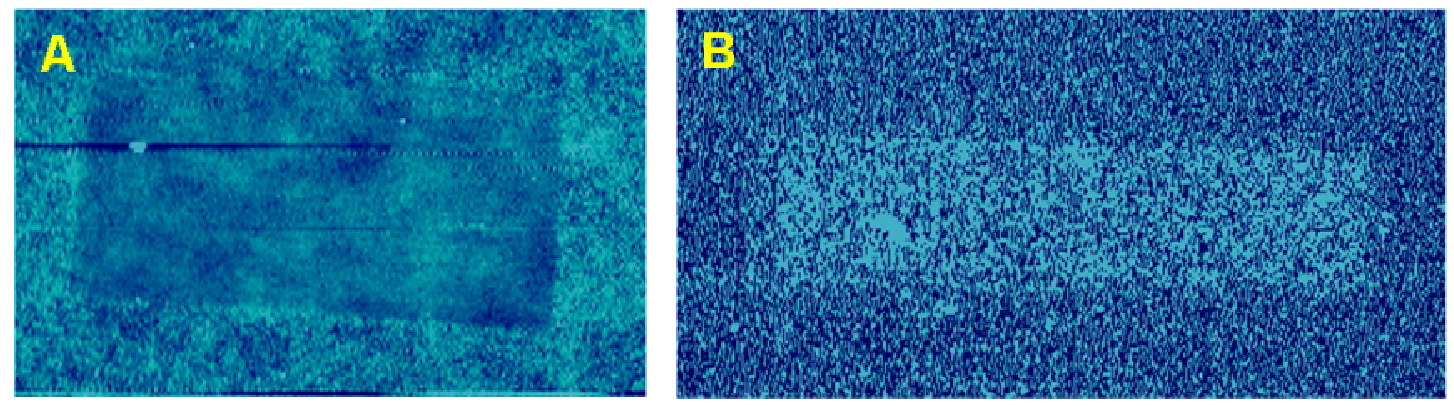

$\mathbf{0}$

$\mu \mathrm{m}$

200

$\mu \mathrm{m}$

14.8

Figure S4. Cy3-streptavidin conjugated patterns imaged with tapping mode AFM after fluorescence had been observed from the written areas. (a) The height of the Cy3-streptavidin patterned regions was roughly $0.4 \mathrm{~nm}$ less than that of the surrounding PEG film, resulting in negative contrast relative to the background in the topographic images. (b) Phase contrast image of another Cy3-streptavidin conjugated pattern. The vertical scale range was $0-1^{\circ}$.

\section{Contact angle measurements of pure water on dry surfaces emulating inked AFM tips:}

Figure S5 shows contact angle measurements of drops of Millipore water on MPTMS-coated glass surfaces that were incubated in $25 \mathrm{mg} / \mathrm{mL}$ maleimide $\mathrm{PEO}_{2}$-biotin and varying amounts of Tween-20 in 
PBS buffer and dried in exactly the same manner as silanized AFM tips. Each data point is the average of 7 trials taken at 10 seconds after depositing the drops at different locations on the model surface. For each point, \pm one standard deviation is indicated by error bars.

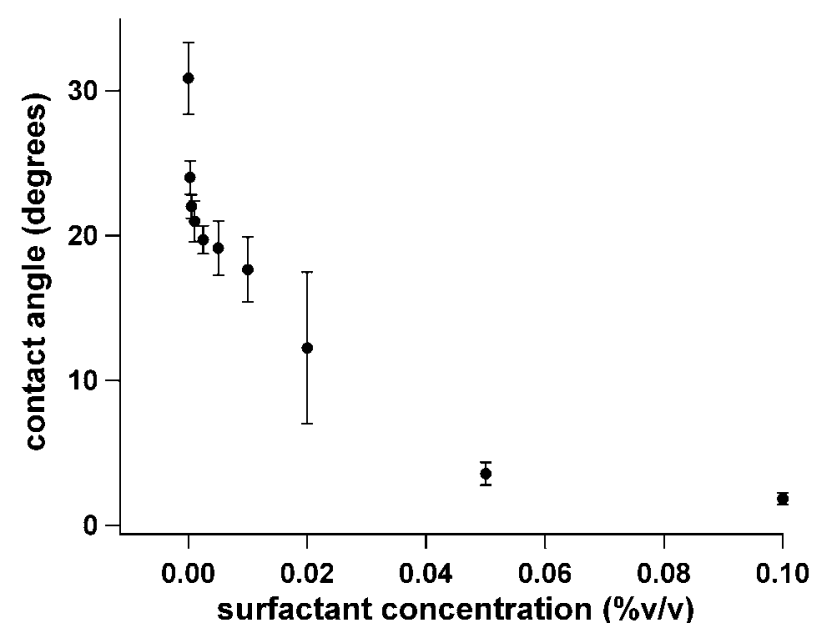

Figure S5. Contact angle measurements of drops of pure water on model surfaces emulating inked AFM tips with biotin and varying amounts of Tween-20.

\section{Determination of liquid-vapor surface tensions of biotin-surfactant solutions using pendant drop}

\section{method:}

The pendant drop technique was used to determine $\gamma_{L V}$ values of biotin solutions. Drops of PBS buffer solutions containing $25 \mathrm{mg} / \mathrm{mL}$ biotin and varying amounts of Tween-20 were suspended from a clean glass capillary (3.02 $\mathrm{mm}$ o.d.). The volume of each drop was approximately $50 \mu \mathrm{L}$. The balance of forces between gravity and the liquid-vapor surface tension of a suspended drop result in a characteristic drop profile, which can be fit with software to give $\gamma_{\mathrm{LV}}{ }^{2}$ Images of suspended drops were captured with a digital camera (Canon PowerShot A75) using a 5x optical telescope and a diffuse bright background after allowing the drops to age in air for 30 seconds. Images were cast into a software-readable format and then input into goccio-synth, version 2.6, an axisymmetric drop shape analysis (ADSA) program available for free at http://infmweb.fi.infn.it/ cf/main.php. The final calculation of $\gamma_{L V}$ required photographic pixels to be converted into physical lengths; the needed scale factor was determined by 
measuring the outer diameter of the glass capillary, both in millimeters with a high-precision caliper and in pixels from the digital photographs. As controls, we compared $\gamma_{\mathrm{LV}}$ values of pure water and hexadecane obtained by this technique with literature values. ${ }^{3}$ The $\gamma_{L V}$ values we obtained agreed with literature values to within $1 \mathrm{~mJ} / \mathrm{m}^{2}$.

\section{References:}

1. Piner, R.D.; Mirkin, C.A. Langmuir 1997, 13, 6864.

2. Busoni, L.; Carlà, M.; Lanzi, L. Rev. Sci. Instrum. 2001, 72, 2784.

3. CRC Handbook of Chemistry and Physics; $79^{\text {th }}$ ed., CRC Press: Boca Raton, 1998. 